\title{
A short proof of a theorem of Kano and Yu on factors in regular graphs
}

\author{
Lutz Volkmann \\ Lehrstuhl II für Mathematik, RWTH Aachen University, 52056 Aachen, Germany \\ e-mail: volkm@math2.rwth-aachen.de \\ Submitted: Jul 13, 2006; Accepted: Jun 1, 2007; Published: Jun 14, 2007 \\ Mathematics Subject Classification: 05C70
}

\begin{abstract}
In this note we present a short proof of the following result, which is a slight extension of a nice 2005 theorem by Kano and Yu. Let $e$ be an edge of an $r$ regular graph $G$. If $G$ has a 1-factor containing $e$ and a 1-factor avoiding $e$, then $G$ has a $k$-factor containing $e$ and a $k$-factor avoiding $e$ for every $k \in\{1,2, \ldots, r-1\}$.
\end{abstract}

Keywords: Regular graph; Regular factor; 1 -factor; $k$-factor.

We consider finite and undirected graphs with vertex set $V(G)$ and edge set $E(G)$, where multiple edges and loops are admissible. A graph is called $r$-regular if every vertex has degree $r$. A $k$-factor $F$ of a graph $G$ is a spanning subgraph of $G$ such that every vertex has degree $k$ in $F$. A classical theorem of Petersen [3] says:

Theorem 1 (Petersen [3] 1891) Every 2p-regular graph can be decomposed into $p$ disjoint 2-factors.

Theorem 2 (Katerinis [2] 1985) Let $p, q, r$ be three odd integers such that $p<q<r$. If a graph has a $p$-factor and an $r$-factor, then it has a $q$-factor.

Using Theorems 1 and 2, Katerinis [2] could prove the next attractive result easily.

Corollary 1 (Katerinis [2] 1985) Let $G$ be an $r$-regular graph. If $G$ has a 1-factor, then $G$ has a $k$-factor for every $k \in\{1,2, \ldots, r\}$.

Proofs of Theorems 1 and 2 as well as of Corollary 1 can also be found in [4]. The next result is also a simple consequence of Theorems 1 and 2 .

Theorem 3 Let $e$ be an edge of an $r$-regular graph $G$ with $r \geq 2$. If $G$ has a 1-factor 
containing $e$ and a 1-factor avoiding $e$, then $G$ has a $k$-factor containing $e$ and a $k$-factor avoiding $e$ for every $k \in\{1,2, \ldots, r-1\}$.

Proof. Let $F$ and $F_{e}$ be two 1-factors of $G$ containing $e$ and avoiding $e$, respectively.

Case 1: Assume that $r=2 m+1$ is odd. According to Theorem 1, the $2 m$-regular graphs $G-E(F)$ and $G-E\left(F_{e}\right)$ can be decomposed into 2-factors. Thus there exist all even regular factors of $G$ containing $e$ or avoiding $e$, respectively. If $F_{2 k}$ is a $2 k$-factor of $G$ containing $e$ or avoiding $e$, then $G-E\left(F_{2 k}\right)$ is a $(2 m+1-2 k)$-factor avoiding $e$ or containing $e$, respectively. Hence the statement is valid in this case.

Case 2: Assume that $r=2 m$ is even. In view of Theorem 1, $G$ has all regular even factors containing $e$ or avoiding $e$, respectively.

Since $G$ has a 1 -factor avoiding $e$, the graph $G-e$ has a 1-factor. In addition, $G-E(F)$ is an $(r-1)$-regular factor of $G$ avoiding $e$, and so $G-e$ has an $(r-1)$-factor. Applying Theorem 2, we deduce that $G-e$ has all regular odd factors between 1 and $r-1$, and these are regular odd factors of $G$ avoiding $e$.

If $F_{2 k+1}$ is a $(2 k+1)$-factor of $G$ avoiding $e$, then $G-E\left(F_{2 k+1}\right)$ is a $(2 m-(2 k+1))$ factor containing $e$, and the proof is complete.

Corollary 2 (Kano and Yu [1] 2005) Let $G$ be a connected $r$-regular graph of even order. If for every edge $e$ of $G, G$ has a 1-factor containing $e$, then $G$ has a $k$-factor containing $e$ and another $k$-factor avoiding $e$ for all integers $k$ with $1 \leq k \leq r-1$.

The following example will show that Theorem 3 is more general than Corollary 2 .

Example Let $G$ consists of 6 vertices $u, v, w, x, y, z$, the edges $u x, v x, w y, z y$, three parallel edges between $u$ and $v$, three parallel edges between $w$ and $z$ and two parallel edges $e$ and $e^{\prime}$ connecting $x$ and $y$. Then $G$ is a 4-regular graph, and $G$ has a 1 -factor containing $e$ and a 1-factor avoiding $e$. According to Theorem $3, G$ has a $k$-factor containing $e$ and a $k$-factor avoiding $e$ for every $k \in\{1,2,3\}$. However, Corollary 2 by Kano and Yu does not work, since the edges $u x, v x, w y$ and $z y$ are not contained in any 1-factor.

\section{References}

[1] M. Kano and Q. Yu, Pan-factorial property in regular graphs, Electron. J. Combin. 12 (2005) N23, 6 pp.

[2] P. Katerinis, Some conditions for the existence of $f$-factors, J. Graph Theory 9 (1985), 513-521.

[3] J. Petersen, Die Theorie der regulären graphs, Acta Math. 15 (1891), 193-220.

[4] L. Volkmann, Graphen an allen Ecken und Kanten, RWTH Aachen 2006, XVI, 377 pp. http://www.math2.rwth-aachen.de/ uebung/GT/graphen1.html. 